\title{
The Effect of Needle Tip Displacement in Co-axial Electrohydrodynamic Processing
}

\author{
Panagiotis Sofokleous $^{1 *}$, Wai K Lau ${ }^{2}$, Mohan Edirisinghe ${ }^{2}$, Eleanor Stride ${ }^{3}$
}

1. Division of Medicine, University College London, Gower street, London, WC1E 6BT, UK

2. Department of Mechanical Engineering, University College London, Torrington Place, London, WC1E 7JE, UK

3. Institute of Biomedical Engineering, Department of Engineering Science, Old Road Campus, University of Oxford, OX3 7DQ, UK

* Corresponding author: Email: panagiotis.sofokleous.10@ucl.ac.uk, Tel: +44 (0) 2076797616. 


\section{ABSTRACT}

Co-axial electrospraying and electrospinning are versatile electrohydrodynamic (EHD) techniques that can be used to encapsulate a variety of materials in the form of polymeric particles and fibres via a one step process. The successful production of uniform encapsulated products in co-axial EHD (CEHD) processing depends on multiple parameters including solution concentration, applied voltage and needle capillary diameter. Although many studies have been conducted to investigate the effects of these parameters, there has been very limited research on how the axial displacement between the two needle tips affects the final products formed. Hence the purpose of this study was to adjust the positioning of the inner needle such that its tip extends beyond, is level with or resides inside that of the outer needle and to thus determine the most effective arrangement for controlling product size, uniformity and/or yield. Core-shell particles were prepared using two polymers, poly(lactic-co-glycolic) acid (PLGA) as the shell and polymethysilsesquioxane (PMSQ) as the core and core-shell fibres using PMSQ as the shell and a volatile liquid, perfluorohexane (PFH) as the core. The products formed were analyzed by optical microscopy, scanning electron microscopy (SEM) and transmission electron microscopy (TEM). After analysis, it was concluded that the most effective arrangement for generating both particles and fibres with the optimal combination of size, uniformity and yield was to have the inner needle $2 \mathrm{~mm}$ inside the outer needle. This allows for formation of a stable cone-jet and successful encapsulation of the inner liquid within the outer liquid, before the outer stable conejet forms. The corresponding collected product diameter and percentage of products in which material was successfully encapsulated were found to be $0.6 \pm 0.1 \mu \mathrm{m}$ and $85 \pm 3 \%$ respectively for particles and $9 \pm 1 \mu \mathrm{m}$ and $92 \pm 2 \%$ for fibres.

KEYWORDS: Electrohydrodynamic; Electrospraying; Electrospinning; Co-axial; Needle; Processing; Forming; Displacement; PLGA; PMSQ; Encapsulation; Particle; Fibre; 


\section{INTRODUCTION}

Electrohydrodynamics is the study of the motion of a conductive liquid in the presence of an external electric field and hence it lies at the interface of electrodynamics ${ }^{1}$ and fluid dynamics ${ }^{2}$. In the electrohydrodynamic process, a liquid is infused into a nozzle and a droplet is formed at the tip of it. When a strong electric field is applied, a charge is induced on the surface of the droplet. If the electric force is strong enough to overcome the surface tension of the liquid, the droplet undergoes different jetting modes depending on the processing parameters ${ }^{3,4}$. The charged jet of liquid is propelled to the collector, which either carries a charge opposite to the droplet or is grounded ${ }^{5,6}$. In the case of a polymer solution, during the flight towards the collector, the solvent evaporates, forming solid products at or before the point of collection. The apparatus used for the bench-top electrohydrodynamic process is relatively cheap and simple to construct. It consists of a DC high voltage electric source with positive or negative polarity, high precision syringe pumps to control the liquid flow to the nozzles, a small diameter nozzle (in the micrometer to millimeter range), tubing to carry the solution from the syringe to the conductive nozzle, a ground or conducting collector and if required, a high speed camera to monitor the process ${ }^{7}$.

Electrospraying and electrospinning are sub-categories of the EHD technique. Both processes transform liquid droplets into micro and nano products when a strong electric field (of the order of several kilovolts) is applied to liquid masses or when a liquid is injected under the effect of an external electric field ${ }^{8}$. The only difference between them is the final product. In electrospraying, the external electric field charges a typically low-viscosity conductive liquid and forces it to disperse into fine droplets ${ }^{9}$. The charged droplets become unstable upon reaching the Rayleigh limit and undergo Coulombic fission, splitting into even smaller ones ${ }^{10}$. Electrospinning is a process by which a high-viscosity solution or melt can be spun into small diameter fibres. Using the electrospinning process, fibres of various diameters and lengths can be produced from almost any soluble polymer and a wide range of ceramic and composite systems ${ }^{11}$. The orientation, porosity and morphology of the fibres is tightly controlled in this process $^{12,13}$. This is important as fibres with small pores and high surface area have applications in tissue engineering, protective clothing, filtration, healthcare, food industry, energy and optical electronics ${ }^{14-17}$. During the fibre production process, the electric force overcomes the surface 
tension of the viscous liquid but the viscoelasticity of the liquid is sufficient to prevent droplet break-up, thus a thin jet is emitted from a liquid cone at the nozzle tip and accelerates towards the collector. The jet is subject to aerodynamic, inertial, rheological and gravitational forces, as well as solvent evaporation. As the diameter of the jet decreases and the surface charge density of the jet increases, the jet shows regular longwave-length instabilities, most notably the electrically driven bending/whipping instabilities ${ }^{18,19}$. The viscosity and the conductivity of the solution have a significant impact on the resulting current and particle/fibre size for both subcategories of $\mathrm{EHD}^{14}$.

Core-shell particles (or macromolecular carriers) and core-shell fibres have potential in emerging scientific themes such as nanomedicine and tissue engineering for encapsulating cells, controlling drug release, forming bubbles for ultrasound diagnostics and treatments, mechanically reinforcing structures for engineering and incorporation of flavours, colours or nutrients for food and drinks ${ }^{7,20,21}$. Micro- and nano-carriers can offer a remarkable solution in delivering passive or active molecules/agents into different target sites. For example Guarino et $a l{ }^{22}$ described the formation of chitosan nanoparticles via the EHD process that can release sodium diclofenac at different $\mathrm{pH}$ conditions. Also many different types of scaffolds such as wound patches, artificial skin, biofilms etc. can be produced by combining co-axial fibres together that can deliver bioactive agents by diffusion or biodegradation mechanisms ${ }^{23}$. Eatemadi et $a l .{ }^{24}$ describes how encapsulated fibres can be used for organ regeneration, such as skin, cartilage and bone regeneration, etc. These scaffolds can be made with natural or synthetic polymer electrospun material or both and can deliver a fixed amount of drug effectively, efficiently and for a defined period of time ${ }^{23}$. For example Li et al. ${ }^{25}$ showed how bioactive silk fibroin/hydroxyapatite (nHAp) fibres can be used to improve bone formation.

Co-axial EHD processing provides a powerful technique for producing particles and fibres on the micro- and nano-scales with a core-shell structure which can encapsulate sensitive core materials (e.g. cells, enzymes or drugs) with a protective shell ${ }^{26-30}$. CEHD uses electrohydrodynamic forces to generate co-axial jets which may contain immiscible liquids or suspensions. For a certain range of values of the applied voltages and flow rates, a cone-jet mode is formed at the tip of the needles. The cone-jet formed in this process has an outer meniscus surrounding an 
inner one. A thread is created from the vertex of each of the two meniscus, giving rise to a stable cone-jet of two co-flowing liquids or suspensions ${ }^{29,31}$.

Electrospun fibres consisting of two separate regions, a core and a shell have been produced by many researchers. Moreover, the core-shell structure can be used for sequential release of drugs for days or even months ${ }^{10,32}$, as the entrapped or encapsulated agents can be released via the shell in a sustained manner. The release rate is proportional to the distribution of the agents and thus, in the case of drug delivery, it is very important to control the distribution of agents or drugs at very small length scales ${ }^{33}$.

Although many research articles have been published in recent years ${ }^{7}$ 21, 26, 34, 35, CEHD processing is a relatively new method and thus, they are still many parameters that need to be investigated. In this work the goal was to use a two needle co-axial system with adjustable needle axial position to study how the displacement between the two needle tips affects the main characteristics and morphology of the core-shell particles and fibres produced. To the best of the authors' knowledge, no other study has systematically varied the needle to needle distance to investigate the effects on product morphology. The percentage number $(n \%)$ of particles $\left(n_{p} \%\right)$ and fibres $\left(n_{f} \%\right)$ in which the material displays a core-shell morphology and is deemed "encapsulated" was investigated in an attempt to determine the most effective height between the two needle tips in order to achieve a small product size and the highest $n \%$ of encapsulated particles and fibres possible using this process.

\section{MATERIALS \& METHODS}

\subsection{Materials}

Poly(lactic-co-glycolic) acid (PLGA) (co-polymer 50:50, Resomer RG503H, molecular weight: $33000 \mathrm{~g} \mathrm{~mol}^{-1}$ ) was provided by Boehringer Ingelheim (Ingelheim, Germany). Acetone and ethanol were obtained from Sigma Aldrich (Poole, UK). Perfluorohexane (PFH) was purchased 
from F2 Chemicals Limited (Lea, UK). Polymethysilsesquioxane (PMSQ) polymer (molecular weight: $7465 \mathrm{~g} \mathrm{~mol}^{-1}$ ) was provided by Wacker Chemie AG, GmbH (Burghausen, Germany). Ethanol was used to prepare PMSQ solutions of $15 \% \mathrm{w} / \mathrm{w}$ and $70 \% \mathrm{w} / \mathrm{w}$ and acetone was used to prepare a PLGA solution of $6 \% \mathrm{w} / \mathrm{w}$ concentration. The solutions were prepared in a volumetric flask by dissolving the polymers in the solvents under constant stirring with magnetic stirrers until the polymers were fully dissolved. All materials were chosen due to their biocompatible properties.

\subsection{Solution Characterization}

The solutions were characterized for their density, surface tension, viscosity and electrical conductivity at a temperature of $25^{\circ} \mathrm{C}$, ambient pressure $(101.3 \mathrm{kPa})$ and a relative humidity of $45-60 \%$. Density was measured using a standard $25 \mathrm{ml}$ density bottle. Using a Kruss tensiometer, the surface tension of the solutions (standard Wilhelmy's plate method) was measured. A UTube glass viscometer suitable for low viscosities (VWR International Ltd, Lutterworth, UK) was used to calculate the viscosity of $6 \% \mathrm{w} / \mathrm{w}$ PLGA and $15 \% \mathrm{w} / \mathrm{w}$ PMSQ solutions. A Brookfield DV-III Ultra Rheometer suitable for small volumes, with an SC4-18 spindle, (Brookfield Viscometers Ltd, Essex, UK) was used to measure the viscosity of the PMSQ solution $70 \% \mathrm{w} / \mathrm{w}$. The electrical conductivity of the solutions was determined using a Jenway $3540 \mathrm{pH} /$ conductivity meter (Bibby Scientific Limited, Staffordshire, UK). All the instruments were calibrated before use according to the manufacturers' instructions. The measured physical properties of the liquids used in the encapsulation experiments are shown in Table I.

\begin{tabular}{|c|c|c|c|c|}
\hline $\begin{array}{c}\text { Material or polymer } \\
\text { solution }\end{array}$ & Density $\left(\mathrm{kg} \mathrm{m}^{-3}\right)$ & $\begin{array}{c}\text { Viscosity } \\
(\mathrm{mPa})\end{array}$ & $\begin{array}{c}\text { Surface Tension } \\
\left(\mathrm{mN} \mathrm{m}^{-1}\right)\end{array}$ & $\begin{array}{c}\text { Electrical Conductivity } \\
\left(\mu \mathrm{S} \mathrm{m}^{-1}\right)\end{array}$ \\
\hline PFH & 1710 & 1.1 & 12 & $<10^{-5}$ \\
\hline PLGA 6\% & 820 & $0.9 \pm 0.2$ & $23.1 \pm 0.5$ & $5.6 \pm 0.2$ \\
\hline PMSQ 15\% & 830 & $1.2 \pm 0.2$ & $23.4 \pm 0.5$ & $0.7 \pm 0.1$ \\
\hline PMSQ 70\% & 970 & $25.4 \pm 0.4$ & $22.1 \pm 0.6$ & $0.8 \pm 0.1$ \\
\hline
\end{tabular}

Table I: Physical properties of liquids used in experiments. All \% refer to weight. The values for PFH were obtained from the manufacturer. 


\subsection{Experimental Setup}

The experimental setup used to form the encapsulated products is shown in Figure 1. It consists of two screw type co-axial brass needles with nylon sealing washers placed between them $(1 \mathrm{~mm}$ thickness each) to control the relative axial displacement of the needles (Figure 2a), which was designed and constructed in previous work ${ }^{36}$. The setup also included two high precision syringe pumps, a high voltage power supply connected to the co-axial needles and a high speed camera (JVC TK-C1481BEG, Weinberger AG, Dietikon, Switzerland) to monitor the process and identify the presence of a cone jet. The outer needle had outer and inner diameters of $1820 \mu \mathrm{m}$ and $1350 \mu \mathrm{m}$, respectively, and the inner needle $560 \mu \mathrm{m}$ and $305 \mu \mathrm{m}$, respectively. High precision syringe pumps ('PHD ultra', Harvard Apparatus Limited, Edenbridge, Kent, UK) were used to regulate the flow rates of the two liquids. Becton-Dickinson (Becton, Dickinson and Company, Oxford, UK) syringes of $10 \mathrm{ml}$ volume capacity containing the solutions were loaded to the syringe pumps. The loaded syringes were then connected to the needle assembly device side entry ports with silicone tubes (outer and inner diameters of $1.5 \mathrm{~mm}$ and $1 \mathrm{~mm}$, respectively). The high voltage power supply (Glassman Europe Limited, Bramley, UK) was connected to the co-axial needle assembly device to provide the electric field between the needle assembly device and the stainless steel jack. A collector (glass containers and glass slides with and without ethanol, respectively) was placed at the top of the jack and connected to the ground electrode. The working distance between the outer needle tip and the collector was set to 120 $\mathrm{mm}$. 

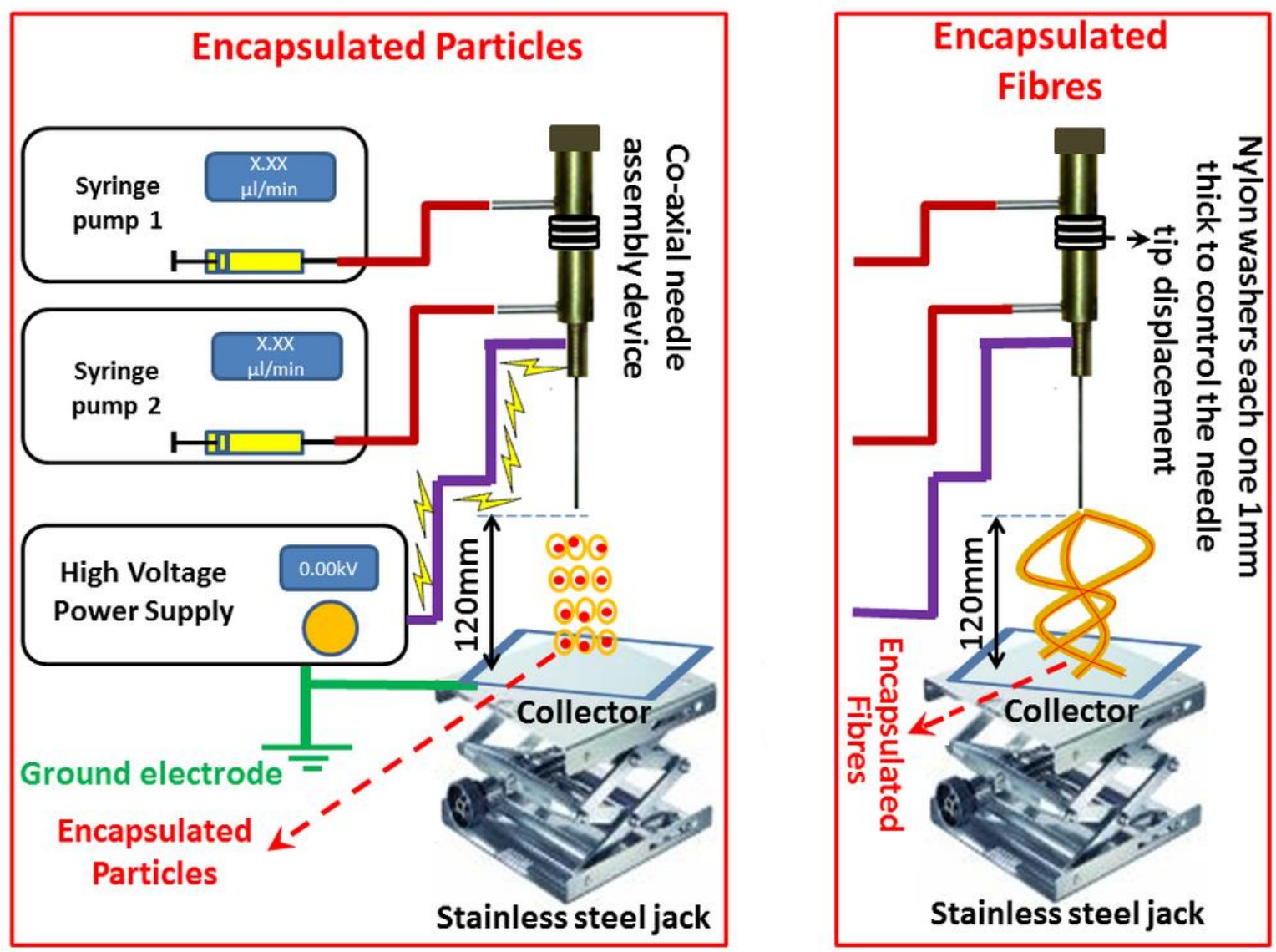

Figure 1: The experimental setup used for the production of encapsulated particles and fibres.

\subsection{Fabrication of Encapsulated Particles and Fibres}

The processing conditions of the CEHD process were adjusted to form uniform encapsulated polymeric particles and fibres. The displacement between the needle tips was adjusted with the help of nylon sealing washers each $1 \mathrm{~mm}$ thick and the two needles were set to be fully concentric (Figure 2b). During the experiments the tip of the inner needle was fixed inside the outer needle at distances of $-4 \mathrm{~mm},-3 \mathrm{~mm},-2 \mathrm{~mm}$ and $-1 \mathrm{~mm}$ relative to the outer needle tip. The tip of the inner needle was also positioned outside of the outer needle at distances of $+3 \mathrm{~mm},+2$ $\mathrm{mm}$ and $+1 \mathrm{~mm}$ (Figure $2 c \& d$ ). In addition the distance between the needle tips was set at the same level $(0 \mathrm{~mm})$. The flow rates for each solution were kept constant during the whole experiment. The applied voltage was adjusted for each displacement during the experiment in order for a stable cone-jet (Taylor cone) to be formed at the needle tip (see Tables II \& III). In this study the effect of applied voltage on particle and fibre characteristics was not examined thoroughly as this parameter was already studied in previous work ${ }^{7,36}$. 


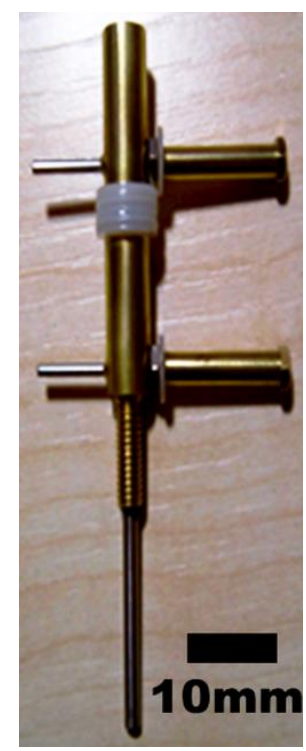

(a)

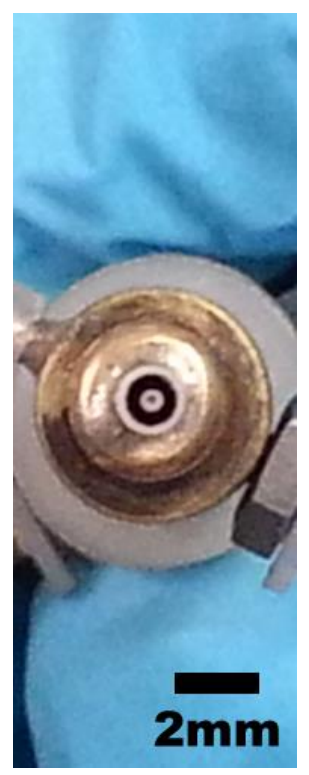

(b)

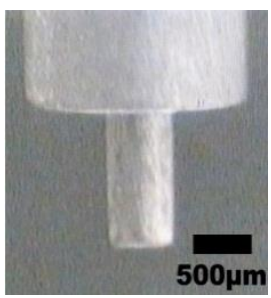

(c)

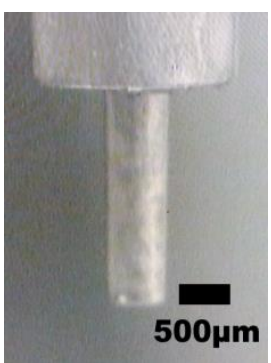

(d)

Figure 2: Images of the needle assembly device with (a) showing the two needle assembly system used and the nylon sealing washers between the needles to control the relative tip displacement, (b) a bottom view of the device showing the concentricity of the two needle tips, (c) and (d) show the inner needle placed by $+1 \mathrm{~mm}$ and $+2 \mathrm{~mm}$ outside the outer needle, respectively.

To form the encapsulated particles the outer needle of the device was infused with $6 \%$ w/w PLGA solution at a flow rate of $12 \mu \mathrm{l} / \mathrm{min}$ and the inner needle was infused with $15 \%$ w/w PMSQ solution at a flow rate of $10 \mu \mathrm{l} / \mathrm{min}$. The particles formed were collected for 300 seconds (for each displacement) in a glass container containing ethanol.

To form encapsulated fibres, the outer needle of the co-axial assembly device was infused with $70 \% \mathrm{w} / \mathrm{w}$ PMSQ solution at a flow rate of $300 \mu \mathrm{l} / \mathrm{min}$ and the inner needle was infused with $\mathrm{PFH}$ at a flow rate of $250 \mu \mathrm{l} / \mathrm{min}$. The fibres were collected for 180 seconds for each displacement on glass slides.

\subsection{Encapsulated Particles and Fibre Characterization}

The encapsulated particle diameter, surface morphology and the percentage of encapsulated particles were studied by scanning electron microscopy (Hitachi S-3400N, SEM) and transmission electron microscopy (Philips CM12 120kV, TEM). The SEM was used to study the 
morphology and size of the encapsulated particles and the TEM was used to identify percentage number of encapsulated particles $n_{p}(\%)$. To study the particles with SEM, they were collected in glass containers containing $10 \mathrm{ml}$ of ethanol and then left to dry for 24 hours (the ethanol was evaporated in $\sim 20-30 \mathrm{~min}$ after collection). Subsequently, they were vacuum-coated with gold for $120 \mathrm{~s}$ and SEM images were obtained at 3-5 kV acceleration voltage. To study the particles with TEM, gold square 75 mesh TEM grids coated with a palladium film were immersed in the ethanol solution after particle collection, then removed and left to dry for 24 hour before examining them. The number of encapsulated particles was calculated according to Equation 1:

$$
n_{p}(\%)=\frac{n_{p e}}{n_{p t}} \times 100
$$

where $n_{p}(\%)$ is the percentage number of encapsulated particles, $n_{p e}$ is the number of encapsulated particles and $n_{p t}$ is the total number of particles examined.

The encapsulated fibres were studied only with optical microscopy (Micropublisher 3.3 RTV, 3.3 megapixel CCD Color-Bayer Mosaic, Real Time Viewing camera, MediaCybernetics, Marlow, UK) as this was sufficient for examining their size and percentage of encapsulated fibres. We were able to identify the PFH liquid droplets in the fibres with the optical microscope, because their density was lower than the solid PMSQ shell surrounding them. All the fibre samples were collected on glass slides and were examined with an optical microscope immediately after production. To further investigate the fibre characteristics, the fibre diameter (A), core/bead diameter (B) and core to core distance (C) were measured by following the same methodology used by Sofokleous et al. ${ }^{7}$. The number of encapsulated fibres was calculated according to Equation 2:

$$
n_{f}(\%)=\frac{n_{f e}}{n_{f t}} \times 100
$$

where $n_{f}(\%)$ is the percentage number of encapsulated fibres, $\mathrm{n}_{f e}$ is the number of encapsulated fibres and $\mathrm{n}_{f t}$ is the total number of fibres examined.

To calculate the percentage numbers $n_{p}(\%)$ and $n_{f}(\%)$ of encapsulated products at least 50-100 different particles or fibres, respectively, were studied. 


\section{RESULTS \& DISCUSSION}

\subsection{Encapsulated Particle formation}

The PMSQ/PLGA particles were produced by keeping the infused flow rates into the needles constant and by adjusting the applied voltage until a stable cone-jet was formed at the outer needle tip. The displacement between the two needles was subsequently varied. This helped to detect the most appropriate height between the needle tips in order to form uniform micro particles and also to get the highest percentage of encapsulated particles $\left(\mathrm{n}_{\mathrm{p}} \%\right)$. Particles were formed due to the high voltage applied to the liquids and because the two conical menisci created at the needle tips were surrounding each other with the PLGA menisci created at the outer needle tip and the PMSQ menisci formed at the inner needle tip. When voltage was applied those menisci formed two-concentric jets which eventually resulted to the formation of particles with the outer liquid encapsulating the inner one (Figure 3$)^{35}$.

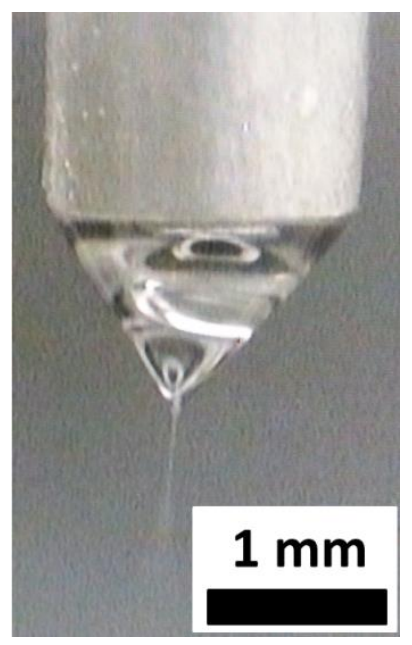

(a)

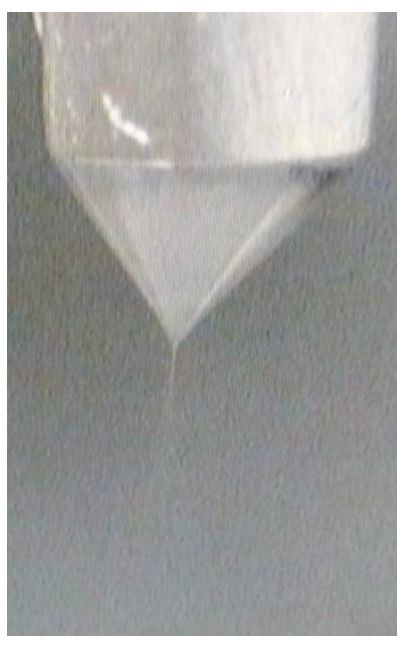

(b)

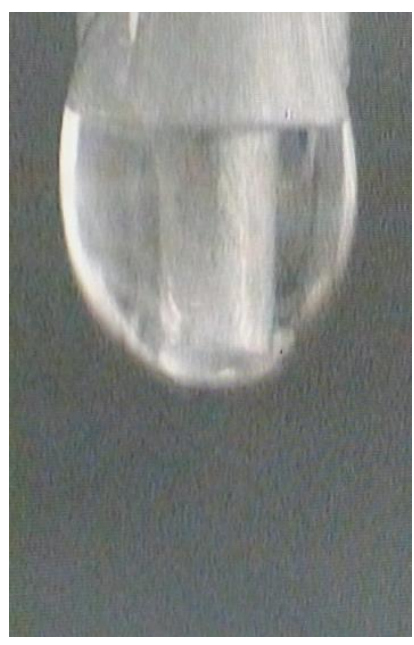

(c)

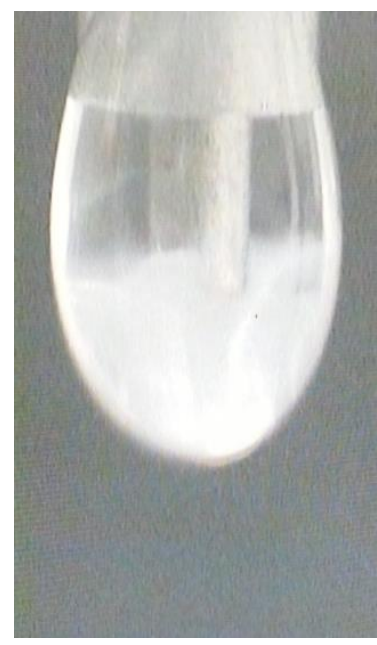

(d) 


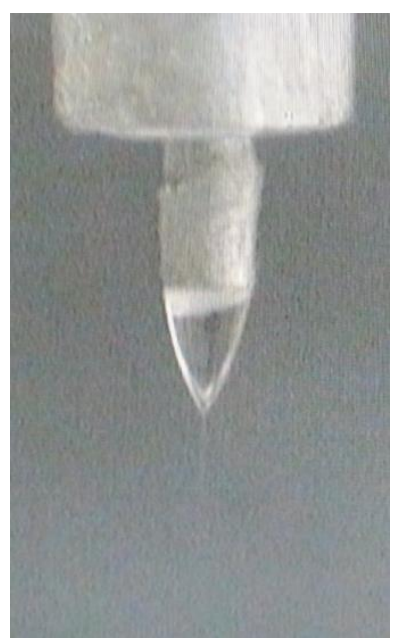

(e)

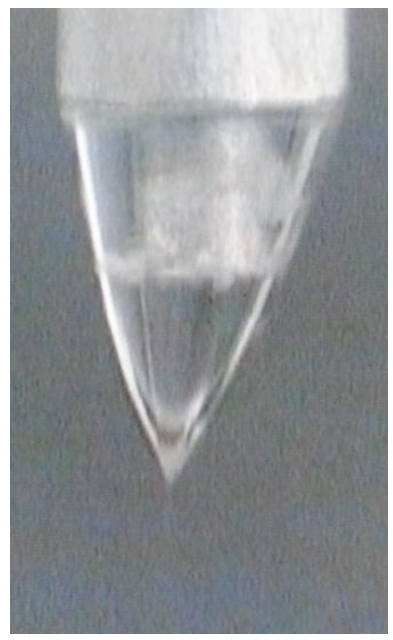

(f)

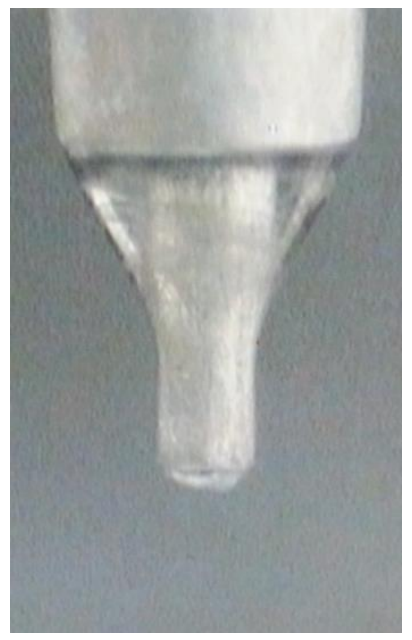

(g)

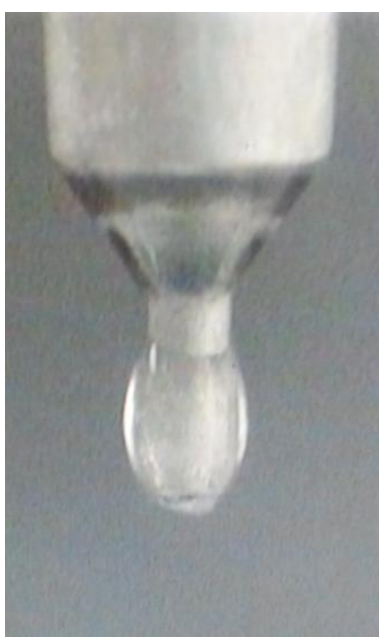

(h)

Figure 3: Cone-jet formation and dripping modes in co-axial EHD processing used for the production of particles at different needle displacements with (a) stable cone-jet formed with no flow in the inner needle at $0 \mathrm{~mm}$, (b) stable cone-jet formed with flow to both needles at $0 \mathrm{~mm}$ showing the outer and inner menisci, (c) dripping mode with no flow in the inner needle at +1 $\mathrm{mm}$, (d) dripping mode with flow to both needles at $+1 \mathrm{~mm}$ demonstrating immiscibility, (e) stable cone-jet formed with no flow in the outer needle at $+1 \mathrm{~mm}$, (f) stable cone-jet formed with flow to both needles at $+1 \mathrm{~mm}$, (g) dripping mode with no flow from the inner needle at $+2 \mathrm{~mm}$ and (h) dripping mode with flow to both needles at $+2 \mathrm{~mm}$. The outer and inner needles shown on the images had an outer diameter of $1820 \mu \mathrm{m}$ and $560 \mu \mathrm{m}$, respectively. Scale bar in (a) applies to all jetting images shown.

The surface morphology of the particles produced was analysed by SEM and TEM. Figure 4 shows SEM images of the particles produced at different needle height displacements and Figure 5 shows TEM images of the encapsulated particles produced at a height of $-2 \mathrm{~mm}$. Table II shows the mean diameter for 100 particles and the percentage $\left(n_{p} \%\right)$ of encapsulated particles achieved at each height.

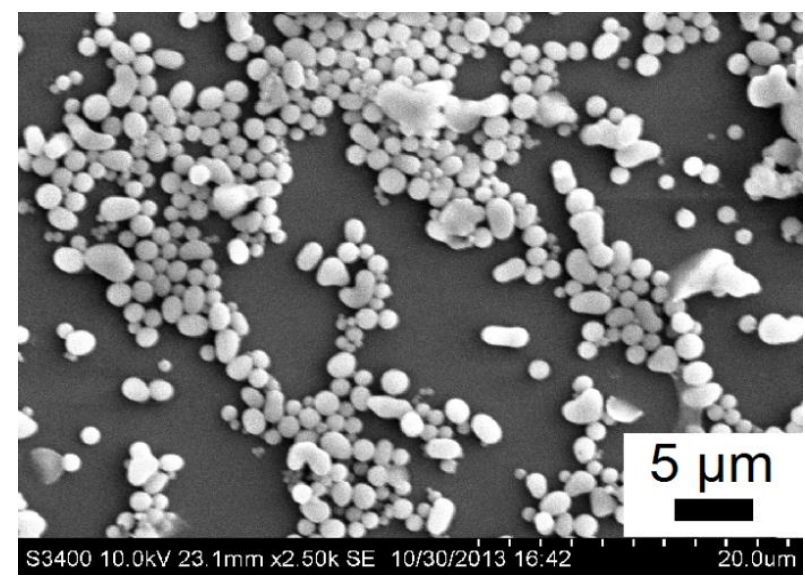

(a)

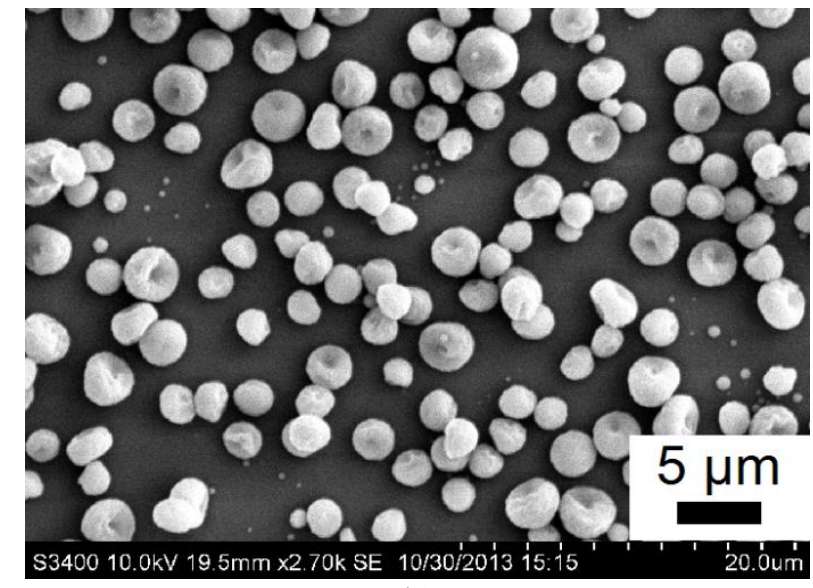

(b) 


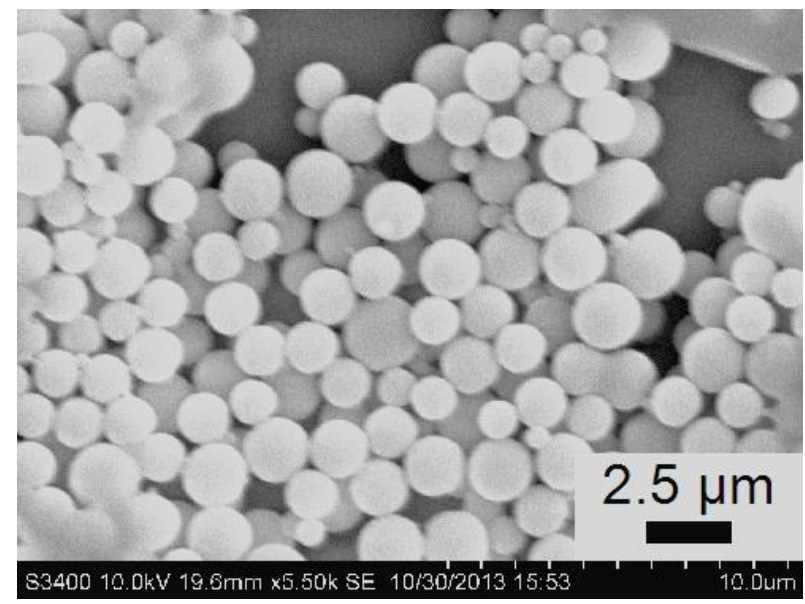

(c)

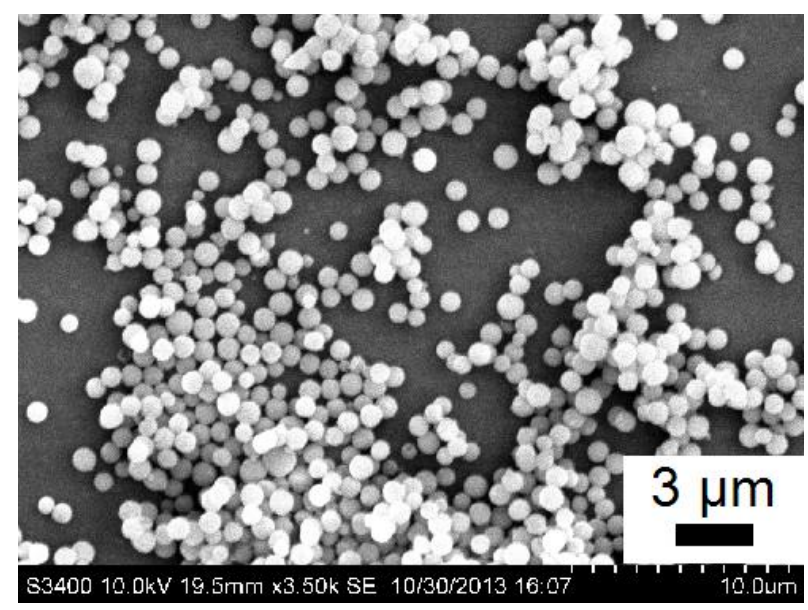

(d)ii

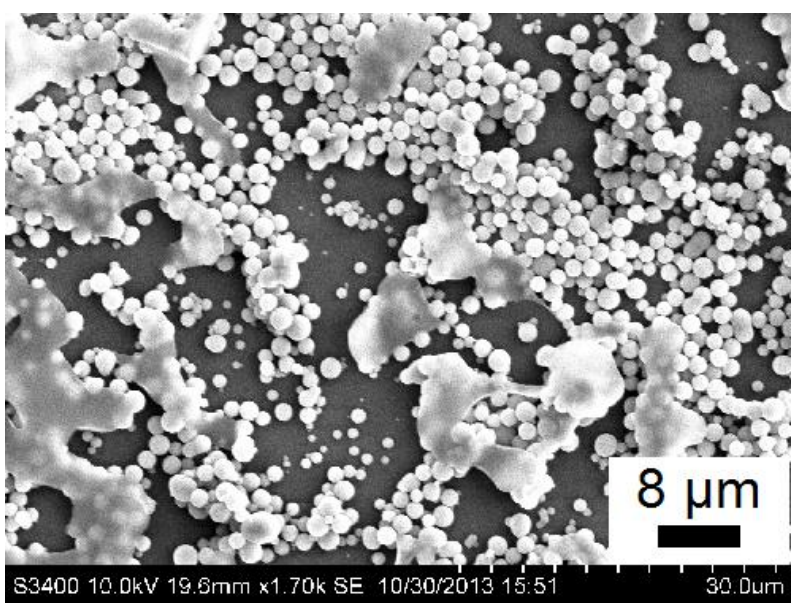

(f)i

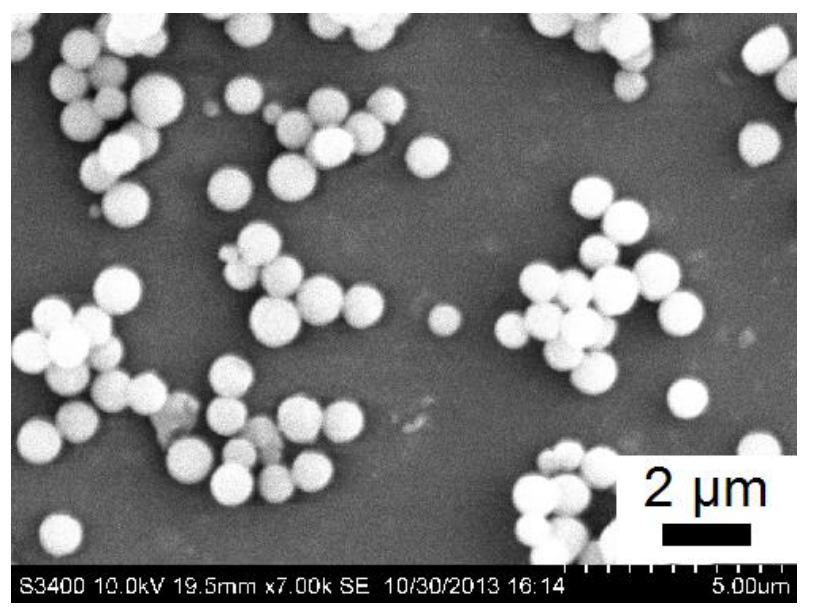

(d)i

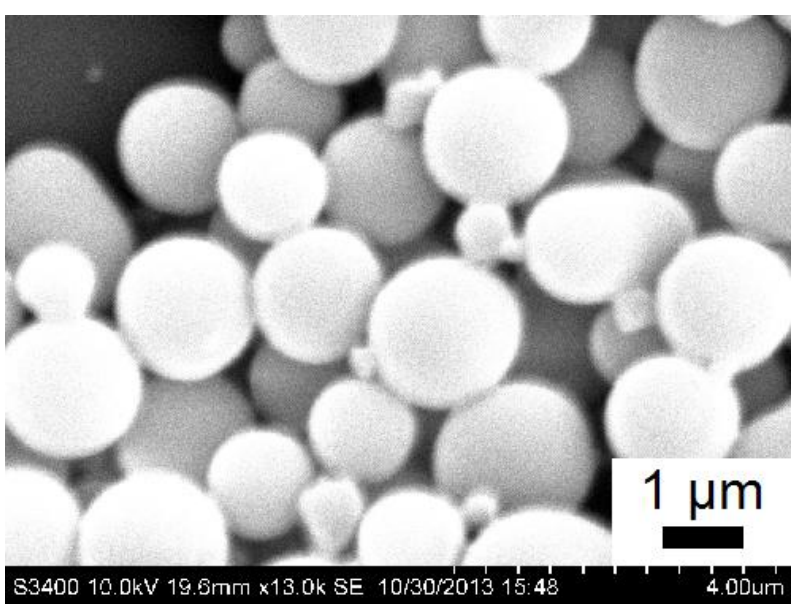

(e)

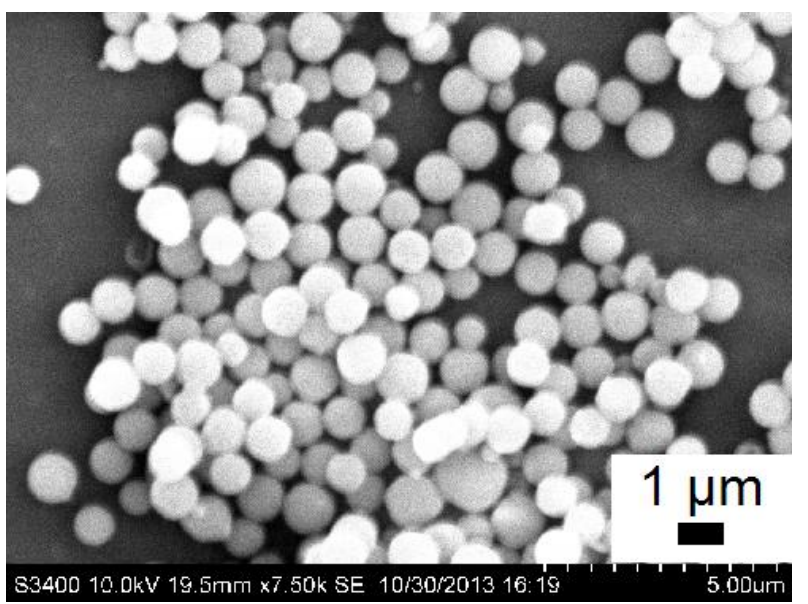

(f)ii

Figure 4: SEM images of particles produced with a relative needle displacement of (a) $+1 \mathrm{~mm}$, (b) $0 \mathrm{~mm}$, (c) $-1 \mathrm{~mm}$, (d)i, ii, $-2 \mathrm{~mm}$, (e) $-3 \mathrm{~mm}$ and (f)i, ii, $-4 \mathrm{~mm}$. 


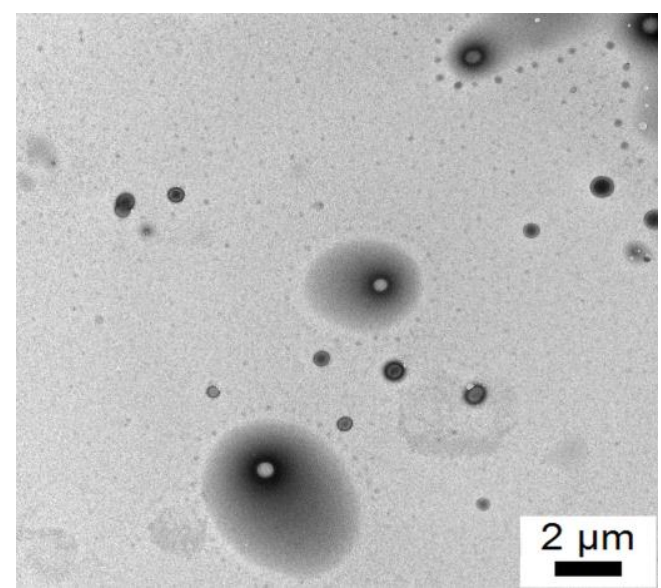

(a)

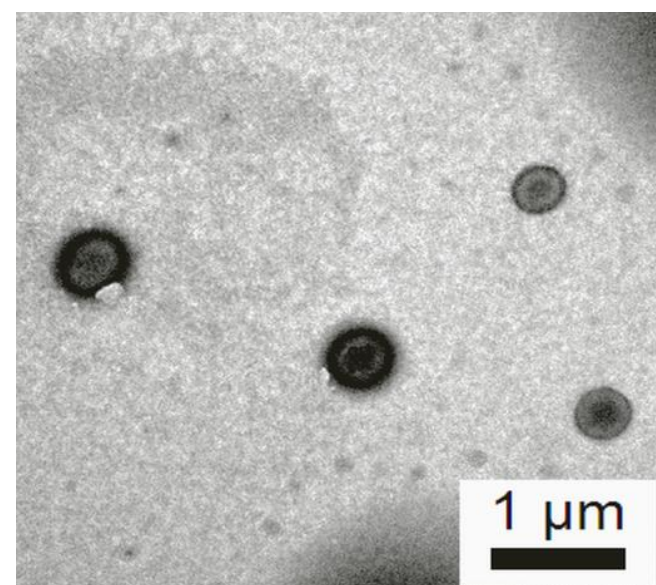

(c)

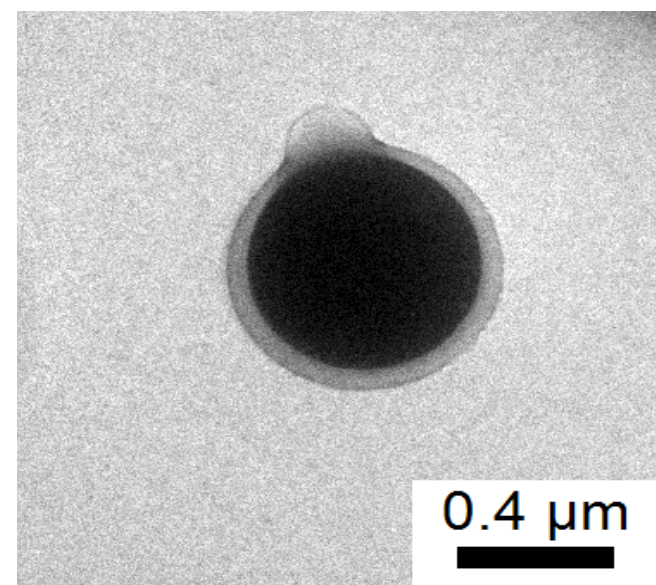

(b)

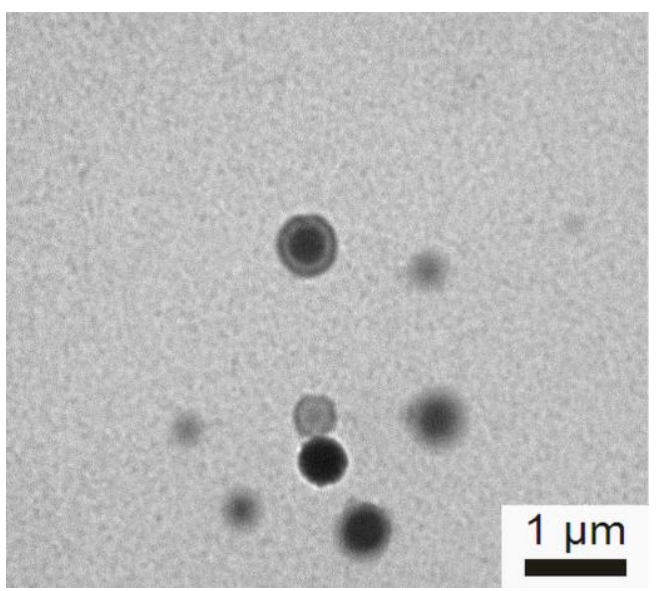

(d)

Figure 5: TEM images of encapsulated particles produced with a relative needle displacement of (a) at $-1 \mathrm{~mm}$, (b) and (c) at $-2 \mathrm{~mm}$ and (d) at $-3 \mathrm{~mm}$.

\begin{tabular}{|c|c|c|c|}
\hline $\begin{array}{c}\text { Relative Needle } \\
\text { Displacement } \\
(\mathrm{mm})\end{array}$ & $\begin{array}{c}\text { Applied Voltage } \\
(\mathrm{kV})\end{array}$ & $\begin{array}{c}\text { Particle } \\
\text { Diameter }(\mu \mathrm{m})\end{array}$ & $\mathrm{n}_{\mathrm{p}} \%$ \\
\hline-4 & 8.8 & $1.3 \pm 0.2$ & $36 \pm 3$ \\
\hline-3 & 9.5 & $1.1 \pm 0.2$ & $42 \pm 3$ \\
\hline-2 & 10.2 & $0.6 \pm 0.1$ & $85 \pm 3$ \\
\hline-1 & 10.9 & $1.3 \pm 0.3$ & $73 \pm 3$ \\
\hline 0 & 12.4 & $2.2 \pm 0.4$ & $60 \pm 3$ \\
\hline+1 & 8.5 & $0.8 \pm 0.1$ & $78 \pm 3$ \\
\hline
\end{tabular}

Table II: Processing parameters used for the production of encapsulated particles and particle characteristics. 
It was noticed that when the inner needle was placed at $+1 \mathrm{~mm}$, the same level $(0 \mathrm{~mm})$ or inside the outer needle, a stable cone-jet could be formed, giving uniform and encapsulated particles, with varying diameters and $n_{p} \%$ values altered at different displacements. Table II shows that the most effective height between the two needle tips in order to achieve the smallest particle size $(0.6 \pm 0.1 \mu \mathrm{m})$ and the highest $n_{p} \%$ value $(85 \pm 3 \%)$ was $-2 \mathrm{~mm}$. Once the inner needle tip was outside the outer needle by more than $+1 \mathrm{~mm}$ then a stable cone-jet could not be formed and hence no uniform or encapsulated particles could be produced. When the needles were at the same level or the inner needle was inside the outer needle the cone-jet formed at the outer needle tip had a length of $\sim 1 \mathrm{~mm}$ (Figure $3 b$ ) and after the inner needle was placed $+1 \mathrm{~mm}$ outside the outer needle then the cone-jet formed from the inner and outer needle tip had a length of $\sim 750$ $\mu \mathrm{m}$ (Figure 3e) and $\sim 2.3 \mathrm{~mm}$ (Figure 3f), respectively. Hence, when the inner needle was positioned $+2 \mathrm{~mm}$ outside the outer needle a cone-jet could not be formed because the inner needle shaft interfered with the menisci formed at the outer needle tip (Figure $3 g \& 3 h$ ).

Table II also shows the applied voltage values used during the experiments in order to achieve a stable cone-jet at each displacement. It was noticed that the lowest applied voltage needed to form a cone-jet corresponded to the inner needle being placed outside the outer needle at a height of $+1 \mathrm{~mm}$. This was due to the liquid in the inner needle behaving as the driving liquid to guide the PLGA solution coming out from the outer needle hence forming a symmetrical stable conejet (Figure 3f). Additionally, with the needle at a height of $+1 \mathrm{~mm}$, the electric field from the inner needle will act tangentially to the liquid meniscus and help with the formation of a stable cone-jet. When the two concentric needles were at the same level, the applied voltage needed to form a stable cone-jet had the highest value. This is due to the competing electric fields from the inner and outer needles. When the distance between the two needle tips was increased to more than $+1 \mathrm{~mm}$ then a cone-jet could not be formed. When the inner needle was placed at different heights inside the outer needle, the applied voltage needed to form the stable cone-jet started to decrease linearly with needle separation $\left(\mathrm{R}^{2}=0.97\right)$ until the displacement was $-4 \mathrm{~mm}$. This can be explained by the relative velocity of the two liquids inside the needle system, the pressure building up in the needle system and the hydrodynamic stresses being applied to the liquids. When the two needles are placed at the same level $(0 \mathrm{~mm})$ the friction being applied to the liquids due to the capillary walls is at maximum value. This results in a decrease in the liquid 
velocity, hence increasing the pressure inside the needle device and causing the hydrodynamic stress to increase ${ }^{37}$. This in turn means a higher electric stress needs to be applied to the liquids that are exiting the needle tips in order to overcome the hydrodynamic stress. The further inside the inner needle is relative to the outer needle, the lower the friction and the hydrodynamic stress and as a result, a lower electric field (applied voltage) is required to get a stable cone-jet ${ }^{38}$.

A relationship between the needle tip displacement and the $n_{p} \%$ value was also observed. The value of $n_{p} \%$ started to increase linearly $\left(\mathrm{R}^{2}=0.999\right)$ when the needle displacement decreased from $0 \mathrm{~mm}$ to $-2 \mathrm{~mm}$. Then it started to decrease in quasilinear manner $\left(\mathrm{R}^{2}=0.854\right)$ when the displacement was decreased from $-2 \mathrm{~mm}$ to $-4 \mathrm{~mm}$. The highest percentage (\%) value of encapsulated particles was observed at a displacement of $-2 \mathrm{~mm}$. The second highest $n_{p} \%$ value was observed at a displacement of $+1 \mathrm{~mm}$. This is expected because, as mentioned before, at that displacement a symmetrical stable cone-jet was obtained while applying the lowest electric force to the liquids. The lowest $n_{p} \%$ value was measured at a displacement of $-4 \mathrm{~mm}$. This is supported by Figure $4(f) i$, which show PMSQ polymer, covering the PLGA particles.

\subsection{Encapsulated Fibre formation}

The PFH/PMSQ fibres were formed by keeping the infused flow rates into the needles constant and by adjusting the applied voltage until a stable cone-jet was formed at the outer needle tip. The displacement between the two needles was also varied in an identical manner to the previous experiments on particles. This again enabled the most appropriate distance between the needle tips to be identified to form uniform microfibres and also to get the highest $n_{f} \%$ value. That was achieved mainly because the PFH was driven by the viscous forces of the thin PMSQ polymer outer shell under the effect of a high electric field causing instantaneous encapsulation of PFH and thus resulting in PMSQ fibres with PFH beads encapsulated in them. PFH can be exchanged with e.g. a drug of choice via a simple diffusion controlled process ${ }^{7}$. Figure 6 shows images of the dripping modes and cone-jet formation in co-axial EHD processing used for the production of PFH encapsulated PMSQ fibres. 


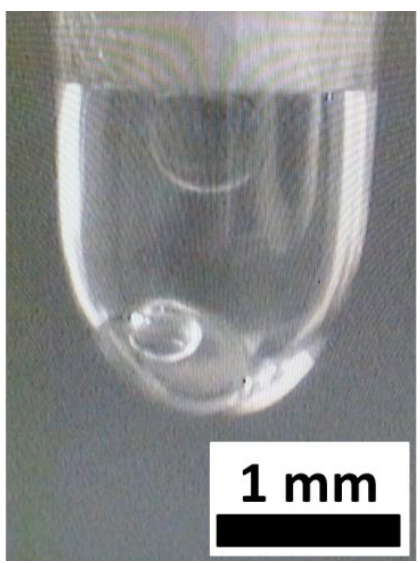

(a)

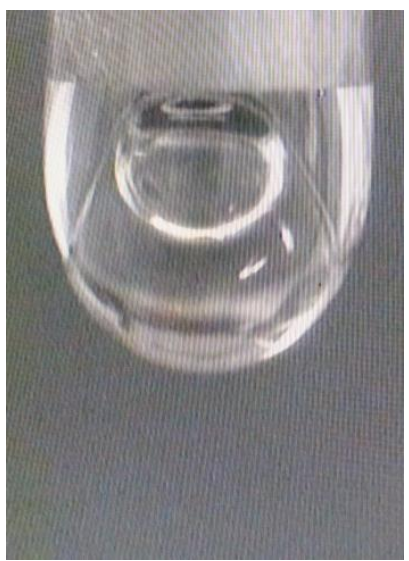

(b)

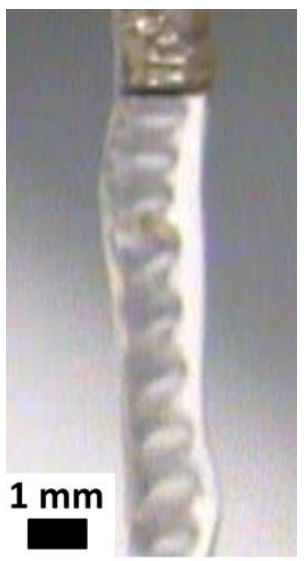

(c)

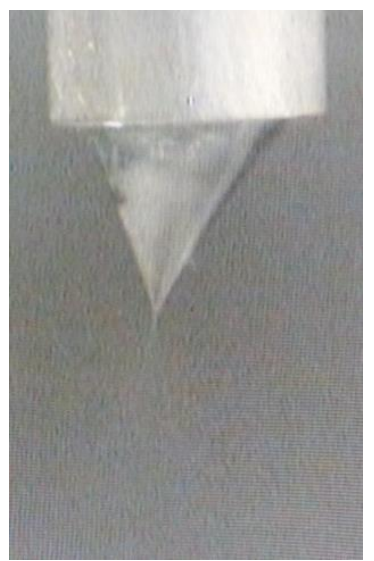

(d)

Figure 6: Dripping modes and cone-jet formation in co-axial EHD processing used for the production of fibres with (a) dripping mode formed with no flow in the inner needle at $0 \mathrm{~mm}$, (b) dripping mode with flow to both needles at $0 \mathrm{~mm}$ demonstrating immiscibility, (c) dripping mode with flow to both needles showing the PFH cores flowing inside the PMSQ polymer and (d) cone-jet formed with flow to both needles at $0 \mathrm{~mm}$. The needle shown on the images had an outer diameter of $1820 \mu \mathrm{m}$.

The surface morphology and encapsulation of the fibres produced were studied via optical microscopy. Figure 7 shows optical micrographs of the fibres produced with different needle displacements. Table III shows the fibres' mean diameter (A), PFH core size (B), PFH core to PFH core distance (C) for 100 fibres (Figure 7a) and the percentage (\%) of encapsulated fibres achieved for each set of conditions.

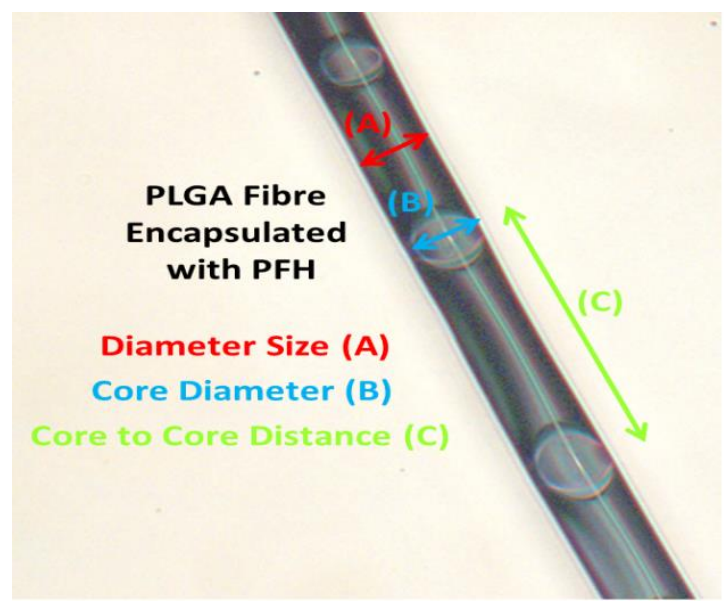

(a)

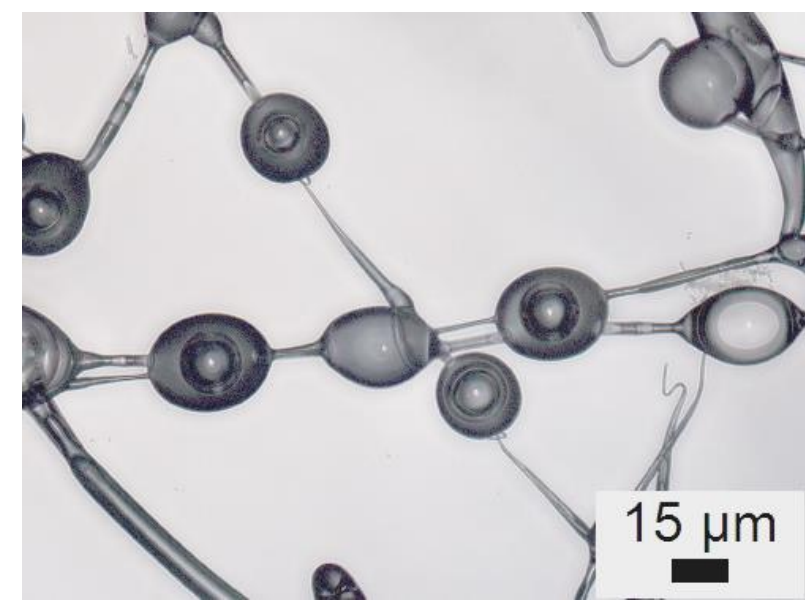

(b) 


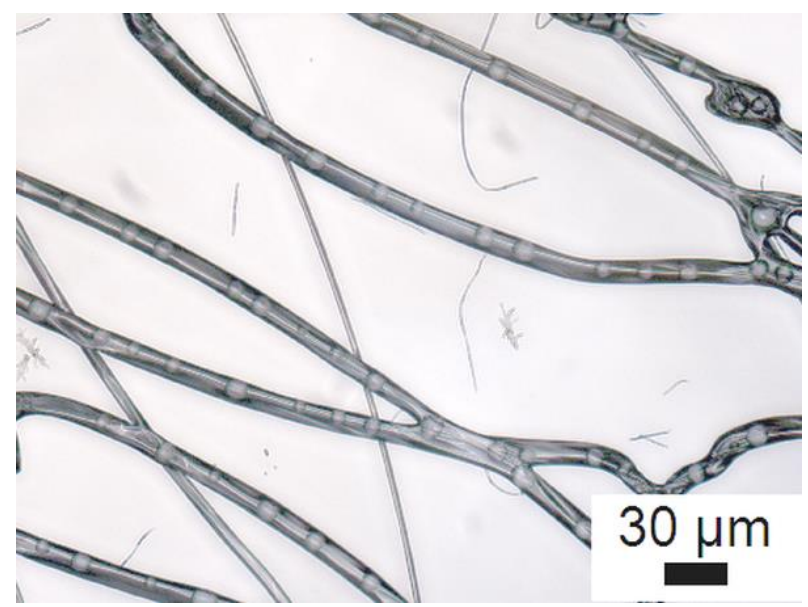

(c)i

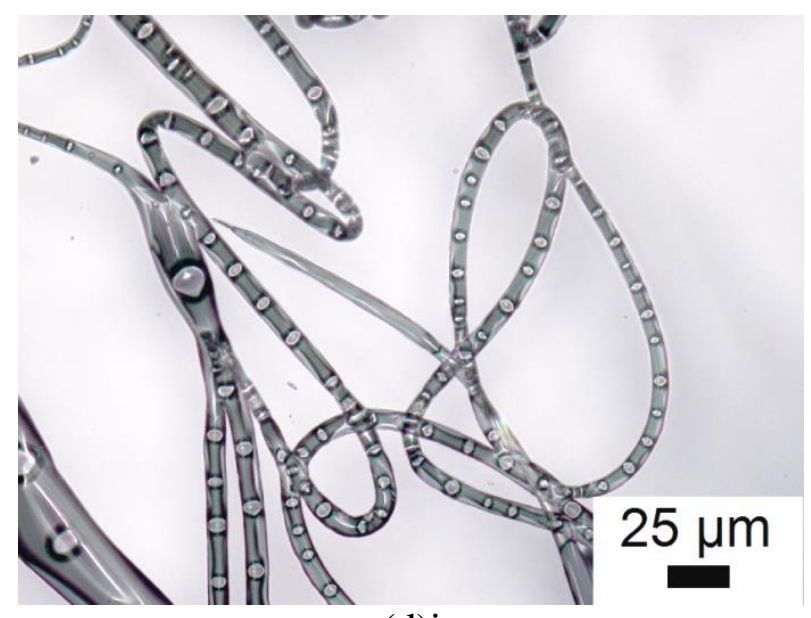

(d) $\mathrm{i}$

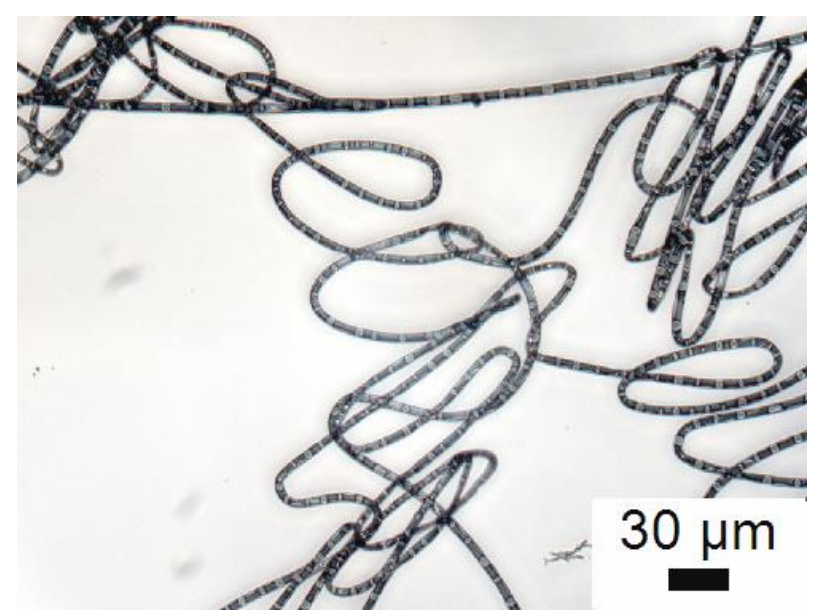

(e)i

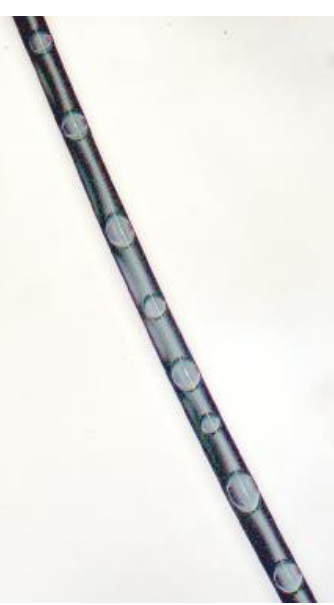

(c)ii

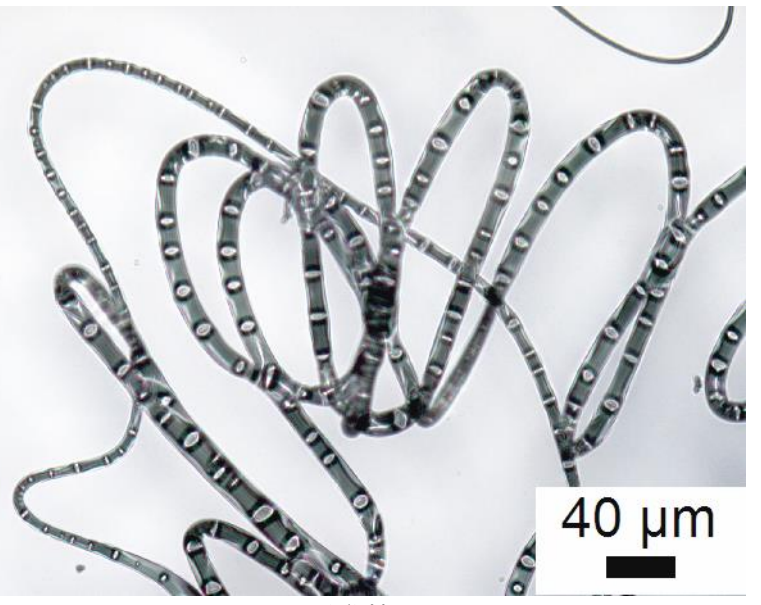

(d)ii

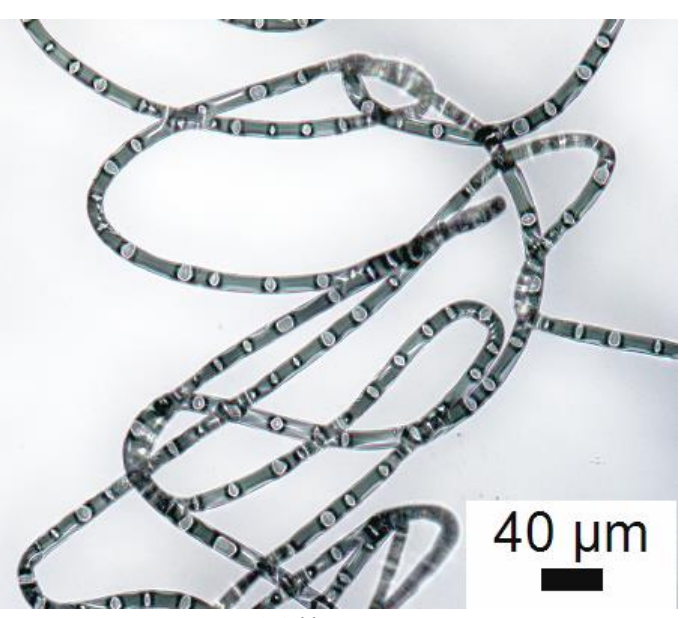

(e)ii 


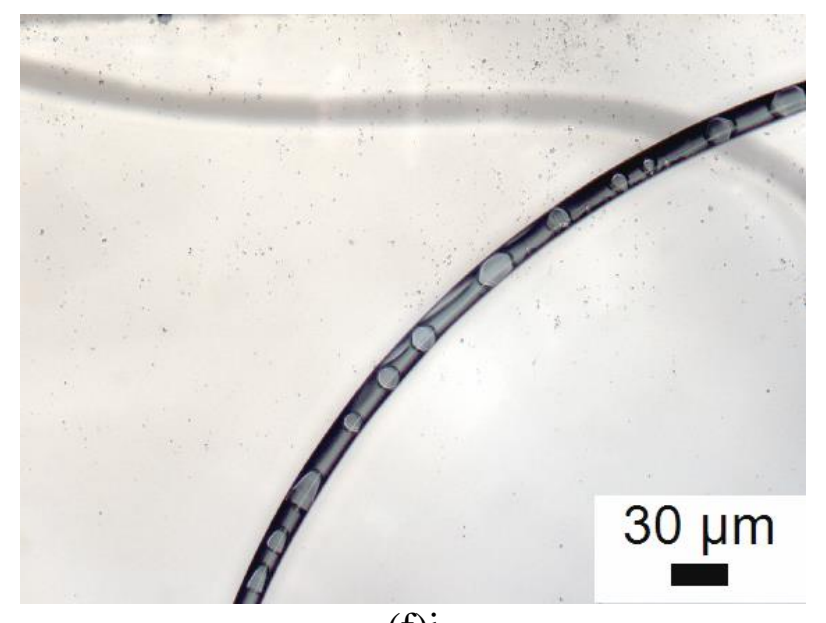

(f)i

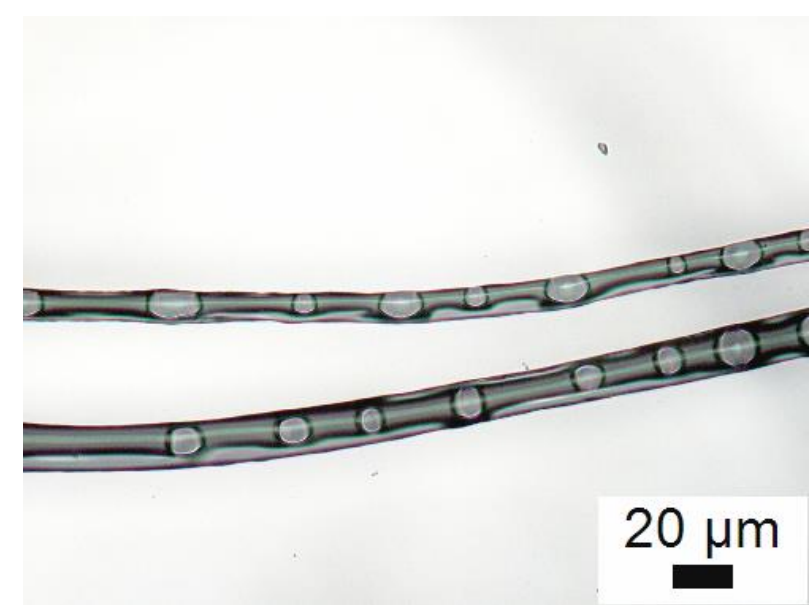

(f)ii

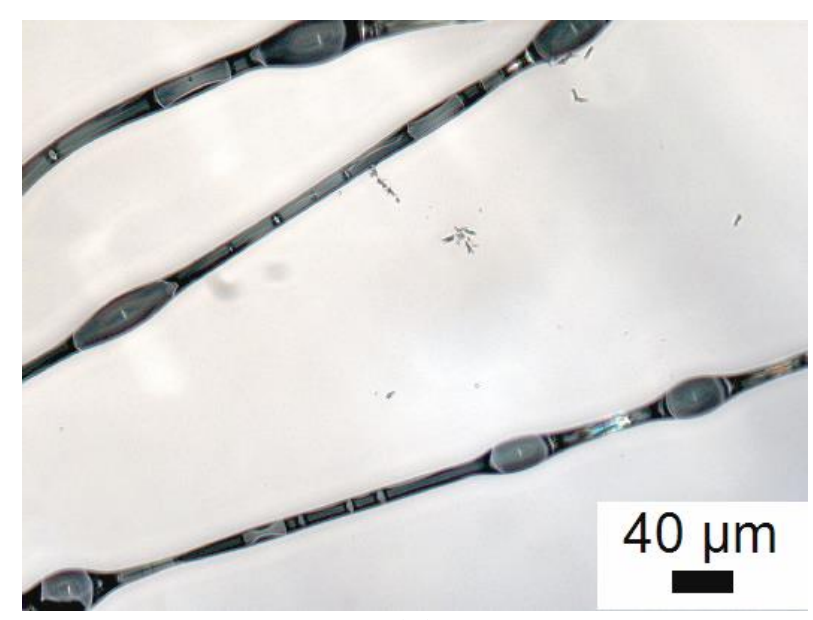

(g)

Figure 7: Image (a) shows the encapsulated fibre characteristics with the rest of them showing optical microscope images of the fibres produced at a needle height displacement of $(\mathrm{b})+1 \mathrm{~mm}$, (c)i, ii, $0 \mathrm{~mm}$, (d)i, ii, -1 mm, (e)i, ii, -2 mm, (f)i, ii, -3 mm and (g) -4 mm.

\begin{tabular}{|c|c|c|c|c|c|}
\hline $\begin{array}{c}\text { Relative } \\
\text { Needle } \\
\text { Displacement } \\
(\mathrm{mm})\end{array}$ & $\begin{array}{c}\text { Applied } \\
\text { Voltage }(\mathrm{kV})\end{array}$ & $\begin{array}{c}\text { Fibre } \\
\text { Diameter } \\
(\mathrm{A}) \text { in } \mu \mathrm{m}\end{array}$ & $\begin{array}{c}\text { Core or } \\
\text { Bead } \\
\text { Diameter } \\
(\mathrm{B}) \text { in } \mu \mathrm{m}\end{array}$ & $\begin{array}{c}\text { Core to } \\
\text { Core } \\
\text { Distance } \\
(\mathrm{C}) \text { in } \mu \mathrm{m}\end{array}$ & $\mathrm{n}_{\mathrm{f}} \%$ \\
\hline-4 & 10.0 & $15 \pm 3$ & $21 \pm 5$ & $27 \pm 8$ & $38 \pm 2$ \\
\hline-3 & 9.3 & $13 \pm 3$ & $11 \pm 3$ & $24 \pm 6$ & $54 \pm 2$ \\
\hline-2 & 9.7 & $9 \pm 1$ & $8 \pm 1$ & $18 \pm 3$ & $92 \pm 2$ \\
\hline-1 & 10.2 & $11 \pm 2$ & $10 \pm 2$ & $21 \pm 5$ & $71 \pm 2$ \\
\hline 0 & 11.6 & $10 \pm 2$ & $9 \pm 2$ & $27 \pm 4$ & $52 \pm 2$ \\
\hline+1 & 13.4 & $4 \pm 2$ & $22 \pm 2$ & $46 \pm 6$ & $23 \pm 2$ \\
\hline
\end{tabular}

Table III: Processing parameters used for the production of encapsulated fibres and fibres characteristics. 
The fibres produced vary from smooth fibres with a gas core pocket found at regular intervals to beaded fibres where the gas is found within the bead. These fibres display a similar morphology to those reported by Diaz et al. ${ }^{39}$. This morphology occurs due to the low viscosity and high surface tension of the inner liquid relative to the outer liquid, which allows the inner droplets to be pulled out at regular intervals producing the morphology displayed in Figure 7.

During the fibre experiments it was noticed that when the inner needle was positioned at the same level $(0 \mathrm{~mm})$ or inside the outer needle by $-1 \mathrm{~mm},-2 \mathrm{~mm}$ and $-3 \mathrm{~mm}$ a stable cone-jet could be formed, giving mostly uniform and encapsulated fibres (Figures $7 c, 7 d$, $7 e$ and $7 f$ ). Similar to the particles' experiment, the fibre diameter and $n_{f} \%$ values were different for each configuration. Table III shows that the most effective height in order to achieve a relatively small fibre diameter $(9 \pm 1 \mu \mathrm{m})$ with the smallest PFH core size $(8 \pm 1 \mu \mathrm{m})$, smallest core to core distance $(18 \pm 3 \mu \mathrm{m})$ and the highest $n_{f} \%$ value $(92 \pm 2 \%)$, is $-2 \mathrm{~mm}$. At a height of $+1 \mathrm{~mm}$ mostly beaded or non-encapsulated (solid) fibres (Figure $7 b$ ) were formed because the jet was not stable. Hence the applied voltage needed to be adjusted repeatedly. Once the inner needle tip was placed outside the outer needle by more than $+1 \mathrm{~mm}$ then a stable cone-jet could not be produced and hence no uniform, beaded or encapsulated fibres were formed. At a height of -4 $\mathrm{mm}$ a stable cone-jet could be obtained, but here too mostly beaded fibres were produced (Figures $7 g$ ). It is likely that this occurred due to misalignment of the needles from the central axis.

Table III also shows the applied voltage values used for fibre production in order to achieve a stable cone-jet at each displacement. It was noticed that when the inner needle was placed outside the outer needle at a height of $+1 \mathrm{~mm}$, the highest voltage had to be applied to achieve a cone-jet which was still not fully stable. The value of $13.4 \pm 1.2 \mathrm{kV}$ reported at that height is an average value of the applied voltage values used to keep the cone-jet stable and preventing it from premature splitting. The high applied voltage and the high viscosity of the solutions used led to increased charge density in the jet. This leads to premature splitting of the jet into multiple fragments at the tip of the cone-jet. After this occurred, the jet is "reset" and resumes the cone-jet mode temporarily ${ }^{13}$. The tip of the cone-jet however, descends a little before being split again. This process is repeated until the jet becomes too long and the polymer solidifies due to the 
solvent evaporation caused mostly by the electric field and the high vapour pressure of ethanol. This leads to a build-up of charge which causes liquid to erupt out of the side of the original cone-jet as a tangent ${ }^{4}$. At a height of $-4 \mathrm{~mm}$ the $n_{f} \%$ value was also very low because the PFH cores were not being surrounded uniformly by the PLGA shell probably due to non-perfectly concentric needles, as in the case of the particles. The core/bead diameter, core to core distance and $n_{f} \%$ are related. A larger core/bead diameter is also associated with a longer core to core distance and a lower $n_{f} \%$ and there seem to be fewer cores with each core containing more core material suggesting it is focused into the core and not distributed uniformly throughout the fibre. It is believed that in the case of $-1 \mathrm{~mm},-2 \mathrm{~mm}$, and $-3 \mathrm{~mm}$, the droplets had little time to decentralise before the electrohydrodynamic forces focuses them to the middle.

When the needle height displacement was decreased from $+1 \mathrm{~mm}$ to $-3 \mathrm{~mm}$ the applied voltage needed to form a stable cone-jet started to decrease in a linear fashion $\left(\mathrm{R}^{2}=0.91\right)$. This can be explained in the same way as for the particles, with the exception of the $+1 \mathrm{~mm}$ height where the high viscosity of the PLGA and PMSQ solutions was the dominant factor. When the displacement was set to $-4 \mathrm{~mm}$ an increase to the applied voltage was needed to get the cone-jet.

Similar to the particle experiments, there was found to be a relationship between the needle tip displacement and the $n_{f} \%$ value which increased linearly $\left(\mathrm{R}^{2}=0.98\right)$ when the needle height displacement decreased from $+1 \mathrm{~mm}$ to $-2 \mathrm{~mm}$. It then started to decrease linearly $\left(\mathrm{R}^{2}=0.95\right)$ when the height was decreased from $-2 \mathrm{~mm}$ to $-4 \mathrm{~mm}$. The highest percentage (\%) value of encapsulated fibres was observed as in the case of particles at the height of $-2 \mathrm{~mm}$. The lowest percentage $(\%)$ was noticed at the heights of $+1 \mathrm{~mm}$ and $-4 \mathrm{~mm}$ for the reasons explained before. As reported previously at those heights mostly beaded fibres were formed.

\subsection{Further Analysis of the Encapsulated Fibres formation}

To compare the experimental parameters and product characteristics of the particles and fibres obtained from the experiments; the applied voltage, product size and percentage ( $n \%)$ of encapsulated products were plotted, respectively, as a function of the needle height displacement

(Figure 8). Figure $8 b$ shows that the diameters of the particles and fibres follow a similar trend 
as needle height displacement is adjusted from $+1 \mathrm{~mm}$ to $-4 \mathrm{~mm}$. Also a similar trend in the percentage of encapsulated particles and fibres can be noticed, with the exception of the results for $+1 \mathrm{~mm}$ displacement. From Figure $8 c$ it can be seen that parameter B increased in size when the needle height displacement decreased from $-2 \mathrm{~mm}$ to $-4 \mathrm{~mm}$. Also parameter $\mathrm{C}$ increased when the height was increased from $-2 \mathrm{~mm}$ to $+1 \mathrm{~mm}$ or decreased $-2 \mathrm{~mm}$ to $-4 \mathrm{~mm}$.

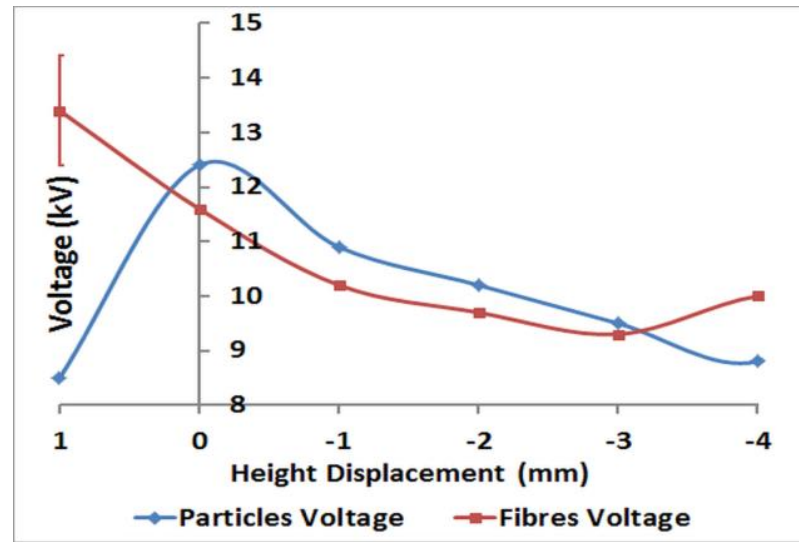

(a)

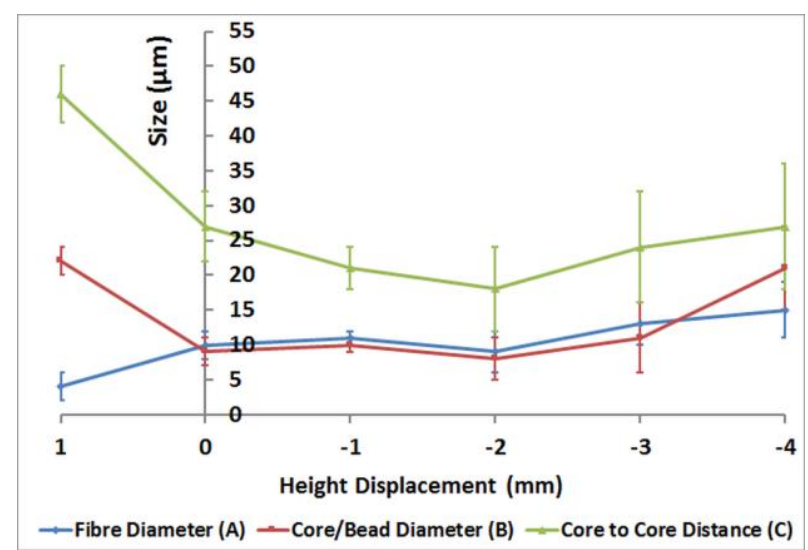

(c)

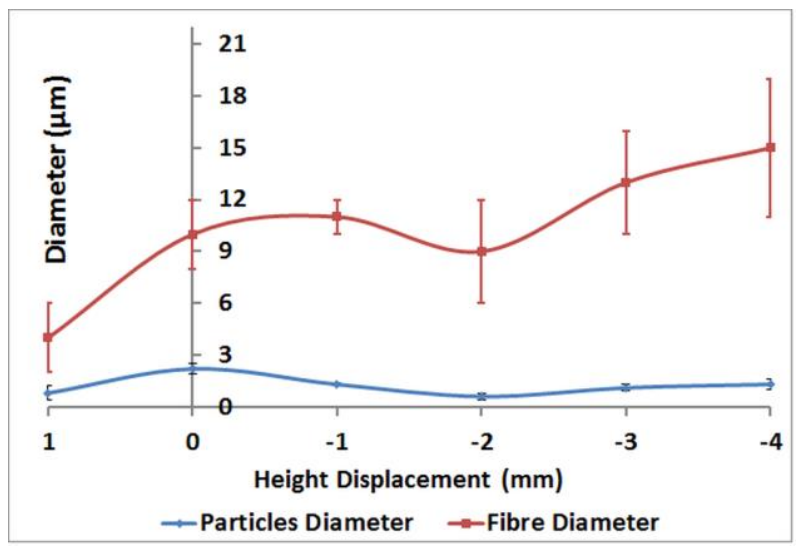

(b)

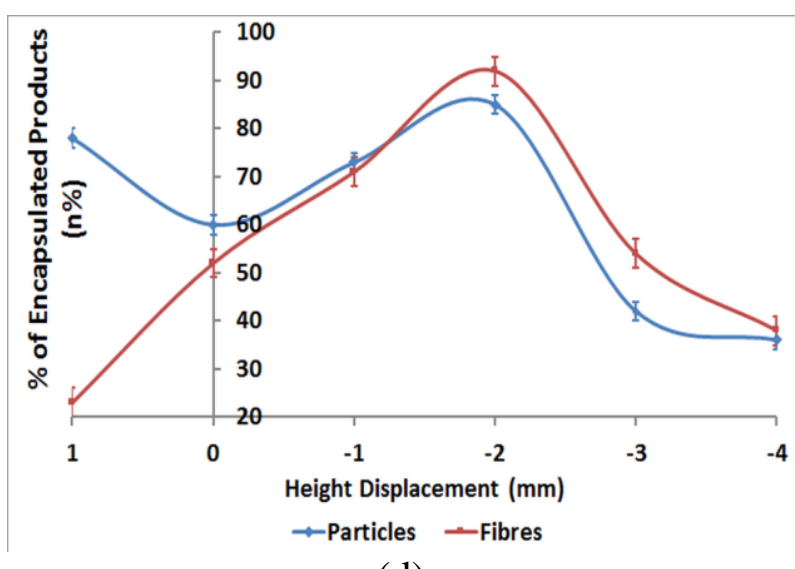

(d)

Figure 8: Key characteristics of the formed products with (a) the applied voltage used to produce the particles and fibres in the stable cone-jet mode as a function of the needle tip displacement, (b) particle and fibre diameters as a function of the needle tip displacement, (c) variation of parameters (A), (B) and (C) of co-axially electrospun PFH encapsulated PMSQ fibres as a function of the needle tip displacement (at a height of $+1 \mathrm{~mm}$ and $-4 \mathrm{~mm}$ only beaded fibres were produced) and (d) the percentage of encapsulated products $(n \%)$ obtained for each displacement. 
One factor of note is that the findings of this research are fully applicable to materials used here but some general observations pertaining to needle placement in CEHDA will be useful in converting EHD science to technological and industrial benefit. Also more work (e.g. Husain et $a l{ }^{40}$ ) is evolving in order to increase the yield in EHD processes, a major industrial turn off to date.

\section{CONCLUSIONS}

Using our custom-made needle device system, this study shows that the relative needle displacement, elevation and end-elevation between the two needle tips significantly affects the main characteristics and the morphology of the encapsulated products formed by co-axial electrohydrodynamic (CEHD) processing. It was found that the proportion of encapsulated products $(n \%)$ changes when the distance between the two needle tips is varied. Furthermore it was demonstrated that by changing the needle displacement, the fibre diameter, the encapsulated PFH core size and core to core distance is altered. Finally it is concluded that the optimum configuration to produce uniform encapsulated micro size particles $(0.6 \pm 0.1 \mu \mathrm{m})$ and fibres $(9 \pm$ $1 \mu \mathrm{m}$ ) with the highest $\mathrm{n} \%$ value was achieved when the inner needle was placed at a displacement of $2 \mathrm{~mm}$ inside the outer needle. At that displacement the percentage of polymethysilsesquioxane (PMSQ) encapsulated poly(lactic-co-glycolic) acid (PLGA) particles was found to be $n_{p} \%=85 \pm 3 \%$ and in the case of perfluorohexane (PFH) encapsulated PMSQ fibres was $n_{f} \%=92 \pm 2 \%$. Also the highest fibre encapsulation efficiency was achieved at that displacement (core to core distance $18 \pm 3 \mu \mathrm{m}$ ).

\section{ACKNOWLEDGEMENTS}

The authors want to thank University College London and the Worshipful Company of Armourers and Brasiers UK for part supporting the doctoral studies of Panagiotis Sofokleous. 


\section{REFERENCES}

1. D. A. Saville, Annual Review of Fluid Mechanics, 1997, 29, $27-64$.

2. J. R. Melcher and G. I. Taylor, Annual Review of Fluid Mechanics, 1969, 1, 111-146.

3. C. N. Ryan, K. L. Smith and J. P. W. Stark, Journal of Applied Physics, 2012, 112, 114510

4. A. Jaworek and A. Krupa, Journal of Aerosol Science, 1999, 30, 873-893.

5. Y. Wu and R. L. Clark, Journal of Biomaterials Science - Polymer Edition, 2008, 19, 573 601.

6. P. D. Noymer and M. Garel, Journal of Aerosol Science, 2000, 31, 1165-1172.

7. P. Sofokleous, M.-W. Chang, B. Y. Ge, E. Stride and M. Edirisinghe, Bioinspired, Biomimetic and Nanobiomaterials, 2011, 1, 48-56.

8. A. Ganan-Calvo, Journal of Fluid Mechanics, 1997, 335, 165-188.

9. U. Stachewicz, J. F. Dijksman, C. U. Yurteri and J. C. M. Marijnissen, Applied Physics Letters, 2007, 91, 254109

10. A. Jaworek and A. T. Sobczyk, Journal of Electrostatics, 2008, 66, 197-219.

11. K. G. Kornev, Journal of Applied Physics, 2011, 110, 124910.

12. B. Sundaray, V. Subramanian, T. S. Natarajan, R.-Z. Xiang, C.-C. Chang and W.-S. Fann, Applied Physics Letters, 2004, 84, 1222-1224.

13. C. J. Luo, S. D. Stoyanov, E. Stride, E. Pelan and M. Edirisinghe, Chemical Society Reviews, 2012, 41, 4708-4735.

14. T. J. Sill and H. A. Von Recum, Biomaterials, 2008, 29, 1989-2006.

15. J. Wei, J. Hu, M. Li, Y. Chen and Y. Chen, RSC Advances, 2014, 4, 28011-28019. 
16. C. J. Luo, S. Loh, E. Stride and M. Edirisinghe, Food and Bioprocess Technology, 2011, 5, 2285-2300.

17. X. Shi, W. Zhou, D. Ma, Q. Ma, D. Bridges, Y. Ma and A. Hu, Journal of Nanomaterials, 2015, 2015, 20.

18. M. M. Hohman, M. Shin, G. Rutledge and M. P. Brenner, Physics of Fluids, 2001, 13, 22012220.

19. A. L. Yarin, S. Koombhongse and D. H. Reneker, Journal of Applied Physics, 2001, 89, 3018-3026.

20. M. Rasekh, K. Nazari, M. Arshad, I. Kucuk, R. Haj-Ahmad, A. Hussain, M. Morris, N. Abbas, M.-W. Chang and X. Li, Current pharmaceutical design, 2016, 22, 2873-2885.

21. M.-W. Chang, E. Stride and M. Edirisinghe, Soft Matter, 2009, 5, 5029-5036.

22. V. Guarino, R. Altobelli and L. Ambrosio, Gels, 2016, 2, 2.

23. D. Li and Y. Xia, Advanced Materials, 2004, 16, 1151-1170.

24. A. Eatemadi, H. Daraee, N. Zarghami, H. Melat Yar and A. Akbarzadeh, Artificial cells, nanomedicine, and biotechnology, 2016, 44, 111-121.

25. C. Li, C. Vepari, H.-J. Jin, H. J. Kim and D. L. Kaplan, Biomaterials, 2006, 27, 3115-3124.

26. M. Enayati, U. Farook, M. Edirisinghe and E. Stride, International Journal of Pharmaceutics, 2011, 404, 110-115.

27. M. Nangrejo, Z. Ahmad and M. Edirisinghe, Ceramics International, 2010, 36, 1217-1223.

28. Y. Wu, Z. Fei, L. J. Lee and B. E. Wyslouzil, Biotechnology and Bioengineering, 2010, 105, 834-841.

29. I. G. Loscertales, A. Barrero, I. Guerrero, R. Cortijo, M. Marquez and A. M. Gañán-Calvo, Science, 2002, 295, 1695-1698. 
30. Z. Sun, E. Zussman, A. L. Yarin, J. H. Wendorff and A. Greiner, Advanced Materials, 2003, 15, 1929-1932.

31. Z. Ahmad, H. B. Zhang, U. Farook, M. Edirisinghe, E. Stride and P. Colombo, Journal of the Royal Society Interface, 2008, 5, 1255-1261.

32. A. Jaworek, Journal of Microencapsulation, 2008, 25, 443-468.

33. W. Cui, Y. Zhou and J. Chang, Science and Technology of Advanced Materials, 2010, 11, 014108.

34. M. W. Chang, E. Stride and M. Edirisinghe, Journal of the Royal Society Interface, 2011, 8, 451-456.

35. J. M. López-Herrera, A. Barrero, A. López, I. G. Loscertales and M. Márquez, Journal of Aerosol Science, 2003, 34, 535-552.

36. P. Sofokleous, E. Stride, W. Bonfield and M. Edirisinghe, Materials science \& engineering. C, Materials for biological applications, 2013, 33, 213-223.

37. I. Marginean, J. S. Page, R. T. Kelly, K. Tang and R. D. Smith, Applied Physics Letters, 2009, 95, 184103.

38. M. N. Reddy and A. Esmaeeli, International Journal of Multiphase Flow, 2009, 35, 10511065 .

39. J. E. Díaz, A. Barrero, M. Márquez and I. G. Loscertales, Advanced Functional Materials, 2006, 16, 2110-2116.

40. O. Husain, W. Lau, M. Edirisinghe and M. Parhizkar, Materials science \& engineering. $C$, Materials for biological applications, 2016, 65, 240-250. 


\section{TABLES LEGEND}

Table I: Physical properties of liquids used in experiments. All \% refer to weight. The values for $\mathrm{PFH}$ were obtained from the manufacturer.

Table II: Processing parameters used for the production of encapsulated particles and particle characteristics.

Table III: Processing parameters used for the production of encapsulated fibres and fibres characteristics. 


\section{FIGURE LEGENDS}

Figure 1: The experimental setup used for the production of encapsulated particles and fibres.

Figure 2: Images of the needle assembly device with (a) showing the two needle assembly system used and the nylon sealing washers between the needles to control the relative tip displacement, (b) a bottom view of the device showing the concentricity of the two needle tips, (c) and (d) show the inner needle placed by $+1 \mathrm{~mm}$ and $+2 \mathrm{~mm}$ outside the outer needle, respectively.

Figure 3: Cone-jet formation and dripping modes in co-axial EHD processing used for the production of particles at different needle displacements with (a) stable cone-jet formed with no flow in the inner needle at $0 \mathrm{~mm}$, (b) stable cone-jet formed with flow to both needles at $0 \mathrm{~mm}$ showing the outer and inner menisci, (c) dripping mode with no flow in the inner needle at $+1 \mathrm{~mm}$, (d) dripping mode with flow to both needles at $+1 \mathrm{~mm}$ demonstrating immiscibility, (e) stable cone-jet formed with no flow in the outer needle at $+1 \mathrm{~mm}$, (f) stable cone-jet formed with flow to both needles at $+1 \mathrm{~mm}$, (g) dripping mode with no flow from the inner needle at $+2 \mathrm{~mm}$ and (h) dripping mode with flow to both needles at $+2 \mathrm{~mm}$. The outer and inner needles shown on the images had an outer diameter of $1820 \mu \mathrm{m}$ and $560 \mu \mathrm{m}$, respectively. Scale bar in (a) applies to all jetting images shown.

Figure 4: SEM images of particles produced with a relative needle displacement of (a) $+1 \mathrm{~mm}$, (b) $0 \mathrm{~mm}$, (c) $-1 \mathrm{~mm}$, (d)i, ii, $-2 \mathrm{~mm}$, (e) $-3 \mathrm{~mm}$ and (f)i, ii, $-4 \mathrm{~mm}$.

Figure 5: TEM images of encapsulated particles produced with a relative needle displacement of (a) at $-1 \mathrm{~mm}$, (b) and (c) at $-2 \mathrm{~mm}$ and (d) at $-3 \mathrm{~mm}$.

Figure 6: Dripping modes and cone-jet formation in co-axial EHD processing used for the production of fibres with (a) dripping mode formed with no flow in the inner needle at $0 \mathrm{~mm}$, (b) dripping mode with flow to both needles at $0 \mathrm{~mm}$ demonstrating immiscibility, (c) dripping mode with flow to both needles showing the PFH cores 
flowing inside the PMSQ polymer and (d) cone-jet formed with flow to both needles at $0 \mathrm{~mm}$. The needle shown on the images had an outer diameter of $1820 \mu \mathrm{m}$.

Figure 7: Image (a) shows the encapsulated fibre characteristics with the rest of them showing optical microscope images of the fibres produced at a needle height displacement of (b) $+1 \mathrm{~mm}$, (c)i, ii, $0 \mathrm{~mm}$, (d)i, ii, -1 mm, (e)i, ii, $-2 \mathrm{~mm}$, (f)i, ii, -3 mm and (g) $-4 \mathrm{~mm}$.

Figure 8: Key characteristics of the formed products with (a) the applied voltage used to produce the particles and fibres in the stable cone-jet mode as a function of the needle tip displacement, (b) particle and fibre diameters as a function of the needle tip displacement, (c) variation of parameters (A), (B) and (C) of co-axially electrospun PFH encapsulated PMSQ fibres as a function of the needle tip displacement (at a height of $+1 \mathrm{~mm}$ and $-4 \mathrm{~mm}$ only beaded fibres were produced) and (d) the percentage of encapsulated products $(n \%)$ obtained for each displacement. 